\title{
ON THE APPROXIMATE SOLUTION OF NONLINEAR SINGULAR INTEGRAL EQUATIONS WITH POSITIVE INDEX
}

\author{
S. M. AMER \\ Department of Mathematics \\ Faculty of Science \\ Zagazig University \\ Egypt \\ (Received March 18, 1994 and in revised form November 28, 1994)
}

\begin{abstract}
This paper is devoted to investigating a class of nonlinear singular integral equations with a positive index on a simple closed smooth Jordan curve by the collocation method. Sufficient conditions are given for the convergence of this method in Holder space.
\end{abstract}

KEY WORDS AND PHRASES. Singular integral equations, collocation method, index of integral equation.

1991 AMS SUBJECT CLASSIFICATION CODES. 45G05, 45L10, 65R20

\section{INTRODUCTION.}

There is a large literature on nonlinear singular integral equations with Hilbert and Cauchy kernel and on related nonlinear Riemann-Hilbert problems for analytic functions, cf. the monograph by Pogorzelski [9] and the other by Guseinov A. I. and Mukhtarov Kh. Sh. [5].

As it is well known, linear singular integral equations of Cauchy type have important applications in hydrodynamics and in the theory of elasticity. Also nonlinear singular integral equations of Cauchy type and related nonlinear Riemann-Hilbert problems are encountered in various problems of continuum mechanics. Many important boundary value problems for partial differential equations of elliptic type can be transformed into the generalized Riemann-Hilbert-Poincare problem, cf. Vekua [11] and Mikhlin et al. [6].

Now, Consider a simple closed smooth Jordan curve $\gamma$ with equation $t=t(s), 0 \leq s \leq \rho$, where s-arc coordinate accounts from fixed point and $\ell$-length of the curve. Dentoe by $\mathrm{D}^{+}$and $\mathrm{D}^{-}$the interior and exterior of $\gamma$ respectively and let the origin be $0 \in \mathrm{D}^{+}$. Denote by $\gamma_{0}$ the unit circle with center at the origin and let $\gamma_{0}^{+}$and $\gamma_{0}^{-}$be the interior and exterior of $\gamma_{0}$ respectively. Consider the conformal mappings $C(w)$ from $\gamma_{0}^{-}$onto $D^{-}$such that $C(\infty)=\infty, \lim _{w \rightarrow \infty} C(w) w^{-1}>0$ and $A(w)$ from $\gamma_{0}^{-}$onto $D^{+}$ such that $A(\infty)=0$.

Consider the following nonlinear singular integral equation (NSIE)

$$
(P(y))(t)=\Psi(t, y(t), B k(t,, y()))=f(t), \quad t \in \gamma
$$

where $\Psi(t, u, v), k(t, \tau, u)$ and $f(t)$ are continuous functions on the domains

$$
\begin{aligned}
& D=\{(t, u, v) ; \quad t \in \gamma, \quad u, v \in(-\infty, \infty)\}, \\
& D_{1}=\{(t, \tau, u,) ; t, \tau \in \gamma, \quad u \in(-\infty, \infty,)\}
\end{aligned}
$$

and on $\gamma$ respectively; The singular integral .

$$
\operatorname{Bk}(t, \tau, y(\tau))=\frac{1}{\pi \mathrm{i}} \int_{\gamma} \frac{\mathrm{k}(\mathrm{t}, \tau, \mathrm{y}(\tau))}{\tau-\mathrm{t}} \mathrm{d} \tau
$$

is a Cauchy principle value and $y(t)$ is unknown function. 
REMARR . The integral Equation (1.1) is equivalent to the following Riemann-Hilbert problem Find a holomorphic function $w(z)=u(z)+i v(z), z=x+i y$, in $\gamma_{0}^{+}$which is continuous in $\gamma_{0}^{+}+\gamma$ and satisfies the boundary condition $\Psi(t, u(t), v(t))=f(t)$ on $\gamma$, (cf. Pogorzelski [9], Wolfersdorf [12]) DEFINITION 1.1 . We denote by $\Phi\left(0, \frac{\ell}{2}\right]$ to be the set of all continuous monotonic increasing functions defined on $\left(0, \frac{\ell}{2}\right.$ ] such that $\lim _{\nu \rightarrow 0} \varphi(\delta)=0$ and $\varphi(\delta) \delta^{-1}$ is nondecreasing function

Consider the Holder space

$$
\mathrm{H}_{\varphi}(\gamma)=\left\{\mathrm{y}(\mathrm{t}) ; \mathrm{t} \in \gamma: \omega(\mathrm{y}, \delta)=\max _{\substack{\left|\mathrm{t}_{1}-\mathrm{t}_{2}\right|<\delta \\ \delta>0}}\left|\mathrm{y}\left(\mathrm{t}_{1}\right)-\mathrm{y}\left(\mathrm{t}_{2}\right)\right|=0(\varphi(\dot{\delta}))\right\}
$$

with the norm

$$
\|y\|_{\varphi}=\|y\|_{C(y)}+\sup _{\delta} \frac{\omega(y, \delta)}{\varphi(\delta)}
$$

where

$$
\|y\|_{C(y)}=\max _{t \in \gamma}|y(t)| \text { and } \varphi(\delta) \text { belongs to } \Phi\left(0, \frac{\ell}{2}\right] .
$$

We denote by $H_{\varphi, 1,1}(D)$ and $H_{\varphi, \varphi_{1}, 1}\left(D_{1}\right)$ to be the spaces of all functions $\Psi(t, u, v)$ and $k(t, \tau, u)$ which satisfy the following conditions:

$$
\begin{aligned}
& \left|\Psi\left(\mathrm{t}_{1}, \mathrm{u}_{1}, \mathrm{v}_{1}\right)-\Psi\left(\mathrm{t}_{2}, \mathrm{u}_{2}, \mathrm{v}_{2}\right)\right| \leq \mathrm{c}_{1}\left\{\varphi\left(\left|\mathrm{t}_{1}-\mathrm{t}_{2}\right|\right)+\left|\mathrm{u}_{1}-\mathrm{u}_{2}\right|+\left|\mathrm{v}_{1}-\mathrm{v}_{2}\right|\right\} \\
& \left|\mathbf{k}\left(\mathrm{t}_{1}, \tau_{1}, \mathrm{u}_{1}\right)-\mathbf{k}\left(\mathrm{t}_{2}, \tau_{2}, \mathrm{u}_{2}\right)\right| \leq \mathrm{c}_{2}\left\{\varphi\left(\left|\mathrm{t}_{1}-\mathrm{t}_{2}\right|\right)+\left(\varphi_{1}\left|\tau_{1}-\tau_{2}\right|\right)+\left|\mathrm{u}_{1}-\mathrm{u}_{2}\right|\right\}
\end{aligned}
$$

respectively, where $\left(t_{1}, u_{1}, v_{1}\right) \in D,\left(t_{1}, \tau_{1}, v_{1}\right) \in D_{1}, t_{1}, \tau_{1} \in \gamma, c_{i}$ are constants, $(i=1,2)$, and $\varphi, \varphi_{1}$ in $\Phi\left(0, \frac{\ell}{2}\right]$.

In the works of Gorlov [4] and Musaev [7], the collocation method is used to find an approximate solution for some classes of NSIE in the Holder space $H_{\alpha}(\gamma)(0<\alpha<1)$.

In the works of Saleh et al. [10], the NSIE (1.1) with positive index $(\chi>0)$ is solved by the Newton Kantorovich method in the subspace:

$$
\Omega_{\varphi}(\gamma)=\left\{\mathrm{y} \in \mathrm{H}_{\varphi}(\gamma) ; \quad \int_{\gamma} \tau^{\mathrm{m}-1} \mathrm{y}(\tau) \mathrm{d} \tau=0, \quad \mathrm{~m}=\overline{1, \chi}\right\}
$$

of the Holder space $\mathrm{H}_{\varphi}(\gamma)$.

In the present paper we shall study the application of collocation method to the solution of NSIE (1.1) with a positive index in Holder space $\mathrm{H}_{\varphi}(\gamma)$.

For this purpose we have to introduce the following:

LEMMA 1.2.(see [10]) Let the functions $\Psi(t, u, v)$ and $k(t, \tau, u)$ belong to $H_{\varphi, 1,1}(D)$ and $H_{\varphi, \varphi_{1}, 1}\left(D_{1}\right)$ respectively, then the operator $P(y)$ has Frechet derivative at any point $y \in H_{\varphi}(\gamma)$ and its derivative has the form :

$$
\left(P^{\prime}(y) h\right)(t)=\Psi_{u}^{\prime}(t, y(t), B k(t ;, y())) h(t)+\Psi_{v}^{\prime}(t, y(t), B k(t, ., y(.))) B\left(k_{u}^{\prime}(t, y()) h(t)\right)
$$

moreover it satisfies Lipschitz condition :

$$
\left\|P^{\prime}\left(y_{1}\right)-P^{\prime}\left(y_{2}\right)\right\|_{\varphi} \leq M\left\|y_{1}-y_{2}\right\|_{\varphi}
$$

in the sphere $S\left(y_{0}, r\right)=\left\|y-y_{0}\right\|_{\varphi} \leq r$, where $M$ is a constant.

The derivative $P^{\prime}(y)$ in (1.4) can be written in the form :

$$
\mathbf{L}_{o} \mathbf{h}=\mathbf{a}(y, t) h(t)+\frac{b(y, t)}{\pi i} \int_{\gamma} \frac{h(\tau)}{\tau-t} d \tau+\frac{c(y, t)}{\pi i} \int_{\gamma} H(t, \tau, y(\tau)) h(\tau) d \tau=f(t)
$$

where;

$$
\begin{aligned}
& a(y, t)=\Psi_{u}^{\prime}\left(t, y_{0}(t), B k\left(t,, y_{0}(.)\right)\right) \\
& b(y, t)=\Psi_{v}^{\prime}\left(t, y_{0}(t), B k\left(t, ., y_{0}(.)\right)\right) k_{u}^{\prime}\left(t, t, y_{0}(t)\right),
\end{aligned}
$$




$$
c(y, t)=\Psi_{v}^{\prime}\left(t, y_{0}(t), B k\left(t, ., y_{0}()\right)\right)
$$

and

$$
H(t, \tau, y(\tau))=\frac{k_{u}^{\prime}\left(t, \tau, y_{0}(\tau)\right)-k_{u}^{\prime}\left(t, t, y_{o}(t)\right)}{\tau-t}
$$

for some initial value $\mathrm{y}_{0} \in \mathrm{H}_{\varphi}(\gamma)$, where, the function $\Psi_{u}^{\prime}$ denotes to the partial derivative of the function $\Psi(\mathrm{t}, \mathrm{u}, \mathrm{v})$ with respect to $\mathrm{u}$

From conditions (1.2), (1.3) and Muskhelishvili [8], see also [5], the follwing lemma is valid

LEMMA 1.3. Let $k(t, \tau, u) \in H_{\left(p, \varphi_{1}, 1\right.}\left(D_{1}\right)$ and $h(t) \in H_{\varphi}(\gamma)$ then the function

$$
\theta(\mathrm{t})=\frac{1}{\pi \mathrm{i}} \int_{\gamma} \frac{\mathrm{k}(\mathrm{t}, \tau, \mathrm{u}(\tau))-\mathrm{k}(\mathrm{t}, \mathrm{t}, \mathrm{u}(\mathrm{t}))}{\tau-\mathrm{t}} \mathrm{h}(\tau) \mathrm{d} \tau
$$

belongs to the space $H_{\varphi}(\gamma)$ and the following inequality is true

$$
\|\theta\|_{\varphi} \leq \mathrm{c}_{3}\|\mathrm{~h}\|_{\varphi}
$$

where $c_{3}$ is a positive constant independent of $h(t)$.

THEOREM 1.4.(see [10]) Let the conditions of Lemma 1.2 be satisfied, $y_{0} \in H_{\varphi}(\gamma)$ and $\left\|L_{\circ}^{-1}\right\|_{\varphi} \leq v_{2}$ and $\left\|L_{0}^{-1} P\left(y_{0}\right)\right\|_{\varphi} \leq \mu_{2}$ then, if $h_{2}=v_{2} M \mu_{2}<\frac{1}{2}$ and $r \geq r_{2}^{0}=\mu_{2}\left(1-\sqrt{\left(1-2 h_{2}\right.}\right) h_{2}^{-1}$, the Equation (1.1) has a unique solution $y^{*}$ in the sphere $S\left(y_{0}, r_{2}^{o}\right)$ to which the modified Newton's method converges with the rate of convergence determined as follows:

$$
\left\|y_{n}-y^{*}\right\|_{\varphi} \leq \frac{\left(1-\sqrt{1-2 h_{2}}\right)^{n}}{\sqrt{1-2 h_{2}}} \mu_{2}
$$

\section{COLLOCATION METHOD.}

Now, we seek an approximate solution of Equation (1.1) in $\Omega_{\varphi}(\gamma)$ in the form :

$$
\mathbf{y}_{\mathbf{n}, \chi}(\eta, t)=\sum_{\mathbf{k}=\mathbf{0}}^{\mathrm{n}-\chi} \eta_{\mathbf{k}} \mathbf{t}^{\mathbf{k}}+\sum_{\mathbf{k}=-\mathbf{n}}^{-\chi-1} \eta_{\mathbf{k}} \mathbf{t}^{\mathbf{k}}, \quad \mathrm{n}>\chi
$$

where the coefficients $\eta_{\mathbf{k}}$ are defined from the system of nonlinear algebraic equations (SNAE) :

$$
\Psi\left(t_{j}, y_{n, \chi}\left(\eta, t_{j}\right), B k\left(t_{j}, ., y_{n, \chi}(\eta, .)\right)\right)=f\left(t_{j}\right)
$$

where

$$
\mathrm{t}_{\mathrm{j}}=\exp \left(\frac{2 \pi \mathrm{i}}{2(\mathrm{n}-\chi)+1} \mathrm{j}\right), \quad \mathrm{j}=\overline{0,2(\mathrm{n}-\chi)} \text {. }
$$

Consider the $(2(n-\chi)+1)$ - dimensional spaces $E_{\varphi}^{(1)}$ and $E_{\varphi}^{(2)}$ with norms

$$
\|\eta\|_{\varphi}^{(1)}=\left\|\mathrm{y}_{\mathbf{n}, \chi}(\eta, .)\right\|_{\varphi}
$$

and

$$
\|\left. u\right|_{\varphi} ^{(2)}=\max \left|u_{j}\right|+\sup _{j \neq k} \frac{\left|u_{j}-u_{k}\right|}{\varphi\left(\left|t_{j}-t_{k}\right|\right)},
$$

respectively, $\mathrm{j}=\overline{0,2(\mathrm{n}-\chi)}$, where

$$
\begin{gathered}
\eta=\left(\eta_{-n}, \ldots, \eta_{-\chi-1}, \eta_{0}, \ldots, \eta_{n-\chi}\right) \in E_{\varphi}^{(1)} \\
u=\left(u_{0}, \ldots, u_{2(n-\chi)}\right) \in E_{\varphi}^{(2)} .
\end{gathered}
$$

Introducing the operator

$$
P_{n}(\eta)=\left(p_{-n+\chi, n}(\eta), \ldots, p_{n-\chi, n}(\eta)\right): E_{\varphi}^{(1)} \rightarrow E_{\varphi}^{(2)}
$$

where

$$
P_{\jmath, n}(\eta)=\Psi\left(t_{j}, y_{n, \chi}\left(\eta, t_{\jmath}\right), B k\left(t_{\jmath}, \cdot y_{n, \chi}(\eta, .)\right)\right), \quad j=-n+\chi, \ldots, n-\chi
$$


We can tewight the SNAL: $(22)$ in the operator form

$$
P_{n}(\eta)=f
$$

where

$$
\mathrm{f}=\mathrm{f}\left(\mathrm{t}_{\mathrm{j}}\right), \quad \mathrm{j}=\overline{0,2(\mathrm{n}-\chi)}
$$

Consider, now the coordinates of the vector $\eta^{(0)}=\left(\eta_{-n}^{(0)}, \ldots, \eta_{-\chi-1}^{(0)}, \eta_{0}^{(0)}, \ldots, \eta_{n-x}^{(0)}\right)$ from $E_{\varphi}^{(1)}$ these are the Fourier coefficients of the function $\mathrm{y}_{0} \in \Omega_{\varphi}(\gamma)$ that is

$$
\eta_{j}^{(0)}=\frac{1}{2 \pi i} \int_{\gamma_{n}} y_{0}(A(w)) w^{-j-1} d w, \quad j=\overline{0, n-\chi}
$$

and

$$
\eta_{\mathrm{j}}^{(0)}=\frac{1}{2 \pi \mathrm{i}} J_{\gamma_{0}} \mathrm{y}_{\mathrm{o}}(\mathrm{C}(\mathrm{w})) \mathrm{w}^{-\mathrm{j}-1} \mathrm{dw}, \quad \mathrm{j}=\overline{-\mathrm{n},-\chi-1} .
$$

Moreover the function $\mathrm{y}_{0} \in \Omega_{\varphi}(\gamma)$ satisfies the conditions of Theorem 14 Analogous to Lemma 1.2, the following lemma is valid.

LEMMA 2.1. Let the conditions of Lemma 1.2 be satisfied, then the operator $P_{n}$ is differentiable in the sense of Frechet at the arbitrary point

$$
x=\left(\eta_{-n}, \ldots, \eta_{-x-1,} \eta_{0}, \ldots, \eta_{n-x}\right) \in E_{\varphi}^{(1)}
$$

moreover,

$$
P_{n}^{\prime}(x) h=\left(P_{-n, n}^{\prime}(x) h, \ldots, P_{-x-1, n}^{\prime}(x) h, P_{o, n}^{\prime}(x) h, \ldots, P_{n-x, n}^{\prime}(x) h\right)
$$

where $h=\left(h_{-n}, \ldots, h_{-\chi-1} h_{0}, \ldots, h_{n-\chi}\right) \in E_{\varphi}^{(1)}$ and

$$
\begin{aligned}
P_{j, n}^{\prime}(x) h= & \Psi_{u}^{\prime}\left(t_{\jmath}, y_{n, \chi}\left(x, t_{\jmath}\right), B k\left(t_{\jmath}, ., y_{n, \chi}(x, .)\right)\right) y_{n, \chi}\left(h, t_{\jmath}\right)+ \\
& \Psi_{v}^{\prime}\left(t_{j}, y_{n, \chi}\left(x, t_{\jmath}\right), B k\left(t_{\jmath}, ., y_{n, \chi}(x, .)\right)\right) B\left(k_{u}^{\prime}\left(t_{\jmath}, ., y_{n, \chi}(x, .)\right) y_{n, \chi}(h, \tau)\right), \quad j \overline{0,2(n-\chi)}
\end{aligned}
$$

The derivative $P_{n}^{\prime}(x)$ satisfies Lipschitz condition in the sphere $S_{\eta(0)}\left(r^{-}\right)$of the space $E_{\varphi}^{(1)}$ :

$$
\left\|\mathbf{P}_{n}^{\prime}\left(\mathbf{x}_{1}\right)-\mathbf{P}_{n}^{\prime}\left(\mathbf{x}_{2}\right)\right\|_{E_{\bullet}^{(2)}} \leq M^{\prime}\left\|x_{1}-x_{2}\right\|_{E_{\bullet}^{(1)}}
$$

where $x_{1}, x_{2} \in S_{\eta(0)}\left(r^{\prime}\right)$ and $M^{\prime}$ is a constant depends on $r^{\prime}, \eta^{(0)}$ and the function $\Psi$.

We shall show that the system of linear algebraic equations (SLAE):

$$
\mathbf{P}_{\mathrm{n}}^{\prime}\left(\eta^{(0)}\right) \mathbf{h}=\mathbf{g}
$$

has the unique solution $h \in E_{\varphi}^{(1)}$ for an abitrary $g=\left(g_{0}, \ldots, g_{2(n-x)}\right) \in F_{4 p}^{(2)}$.

For this aim, we consider the SALE:

$$
\begin{aligned}
a\left(y_{0}, t_{j}\right) y_{n, \chi}\left(h, t_{j}\right) & +\frac{b\left(y_{o}, t_{j}\right)}{\pi i} \int_{\gamma} \frac{y_{n, \chi}(h, \tau)}{\tau-t_{j}} d \tau+ \\
& +\frac{c\left(y_{0}, t_{j}\right)}{\pi i} \int_{\gamma} H\left(t_{j}, \tau, y_{0}\right) y_{n, \chi}(h, \tau) d \tau=g\left(t_{j}\right), \quad j=\overline{0,2(n-\chi)}
\end{aligned}
$$

corresponding to (by collocation method ) the singular integral equation

$$
a\left(y_{0}, t\right) y(t)+\frac{b\left(y_{o}, t\right)}{\pi i}-\int_{\gamma} \frac{y_{(\tau)}}{\tau-t} d \tau+\frac{c\left(y_{0}, t\right)}{\pi i} \int_{\gamma} H\left(t, \tau, y_{0}(\tau)\right) y(\tau) d \tau=g(t)
$$

According to the collocation method, we seek an approximate solution of Equation (1.5) in $\Omega_{\varphi}(\gamma)$ in the form :

$$
h_{n, \chi}(t)=\sum_{k=-n}^{n-\chi} \beta_{k} t^{k}, \quad t \in \gamma
$$

where the coefficients $\beta_{k}$ are defined from the SLAE : 


$$
\begin{aligned}
\left(a\left(y, t_{j}\right)\right. & \left.+b\left(y, t_{j}\right)\right) \sum_{k=0}^{n-x} \beta_{k} t_{j}^{h}+\left(a\left(y, t_{\jmath}\right)-b\left(y, t_{j}\right)\right) \sum_{k=-n}^{-x-1} \beta_{h} t_{j}{ }^{k}+ \\
& +\frac{c\left(y, t_{j}\right)}{\pi i} \int_{\gamma} H\left(t_{j}, \tau, y(\tau)\right) \sum_{h=-n}^{n-\chi} \beta_{k} \tau^{h} d \tau=f\left(t_{\jmath}\right), \quad j=0,2(n-\chi)
\end{aligned}
$$

THEOREM 2.2. Let $a(y, t), b(y, t)$, and $f(t)$ (by all arguments) belong to $H_{i p}(\gamma), a^{2}(y, t)-b^{2}(y, t) \neq 0$, on $\gamma$ the index $\chi=\operatorname{ind}(a(y, t)+b(y, t))>0$ and the operator $P^{\prime}(\gamma)$ has a lincan incese in $H_{\varphi}(\gamma)$. then for all $\mathrm{n} \geq \max \left(\mathrm{n}_{0}, \chi\right)$,

$$
\mathrm{n}_{\mathrm{o}}=\min \left\{\mathrm{n} \in \mathbb{I N}: \mathrm{e}_{\mathrm{n}}=\mathrm{d}_{1} \varphi\left(\frac{1}{\mathrm{n}-\chi}\right) \ln (\mathrm{n}-\chi)<1\right\},
$$

the system (2.8) has the unique solution $\left\{\beta_{\mathrm{k}}^{*}\right\}_{\mathrm{k}=-\mathrm{n}}^{\mathrm{n}-\chi}$ and the approximate solution

$$
\mathrm{h}_{\mathrm{n}, \mathrm{x}}^{*}=\sum_{\mathrm{k}=\mathrm{n}}^{\mathrm{n}-\mathrm{x}} \beta_{\mathrm{k}}^{*} \mathrm{t}^{\mathrm{k}}, \quad \mathrm{t} \in \gamma .
$$

of Equation (1.5) converges to its exact solution $\mathrm{h}^{*}$, moreover

$$
\left\|h^{*}(t)-h_{u, \chi}^{*}(t)\right\|_{\varphi} \leq d_{2} \varphi\left(\frac{1}{n-\chi}\right) \ln (n-\chi) .
$$

Here and below $d_{1} d_{2}, \ldots$ are constants do not depend on $n$.

PROOF. From Gakhov [3] and [5,8] we shall write equation $(1.5)$ in $\Omega_{\varphi}(\gamma)$ in th form.

$$
\mathbf{L h}=\mathbf{E h}+\mathbf{G h}=\mathbf{q}
$$

where

$$
\begin{gathered}
(E h)(t)=\psi^{-}(t) h^{+}(t)-\psi^{+} t^{x} h^{-}(t), \\
(G h)(t)=\frac{Q(t) c(y, t)}{\pi i} \int_{\gamma} H(t, \tau, y(\tau)) h(\tau) d \tau, \\
Q(t)=\frac{\psi^{-}(t)}{a(y, t)+b(y, t)}, \quad q(t)=Q(t) f(t), \\
\psi^{+}(t)(a(y, t)+b(y, t))=\psi^{-}(t) t^{-x}(a(y, t)-b(y, t)), \\
\psi(z)=\exp (\Gamma(z)) \quad \text { and } \quad \Gamma(z)=\frac{1}{\pi i} \int_{\gamma} \ln \left[\tau^{-x} \frac{a(y, \tau)-b(y, \tau)}{a(y, \tau)+b(y, \tau)}\right] \frac{d \tau}{\tau-z} .
\end{gathered}
$$

Moreover, $E$ is linear and $G$ is completely continuous from $\Omega_{\varphi}(\gamma)$ into $H_{\varphi}(\gamma)$. Denote by $X_{n, \chi}=\left\{h_{n, \chi}=h_{n, \chi}^{+}-h_{n, \chi}^{-}\right\}$to be the $(2(n-\chi)+1)$-dimensional subspace of the space $\Omega_{p}(\gamma)$ where

$$
\mathrm{h}_{\mathrm{n}, \chi}^{+}=\sum_{\mathrm{k}=0}^{\mathrm{n}-\chi} \beta_{\mathrm{k}} \mathrm{t}^{\mathrm{k}} \quad, \quad \mathrm{h}_{\mathrm{n}, \chi}^{-}=-\sum_{\mathrm{k}=-\mathrm{n}}^{-\chi-1} \beta_{\mathrm{h}} \mathrm{t}^{\mathrm{h}}
$$

Let $P_{n, \chi}$ be the projection operator into the set of interpolation polynomials of degree $n-\chi$ with respect to the collocation points $t_{j}, j=\overline{0,2(n-\chi)}$, then the system (2.8) can be written in $X_{n, \chi}$ as a linear operator

$$
L_{i, x}, h_{n, x}=E_{n, x} h_{n, x},+G_{n, x} h_{n, x}=q_{n, x}
$$

where

$$
E_{n, \chi} h_{n, \chi}=P_{n, \chi} E h_{n, \chi}, \quad G_{n, \chi} h_{n, \chi}=P_{n, \chi} G h_{n, \chi} \quad \text { and } \quad q_{n, \chi}=P_{n, \chi} q .
$$

Now, we determine the difference: $\operatorname{Lh}_{n, x}-L_{n, x}, h_{n, x} \in X_{n, x}$ in $H_{\varphi}(\gamma)$ from (2.9)-(2.11) we have

$$
\begin{aligned}
& L h_{n, x}-L_{n, x} h_{n, x}=\left(\psi^{-} h_{n x}^{+}-\psi^{+} t^{x} h_{n, x}^{-}\right)-P_{n x}\left(\psi^{-} h_{n x}^{+}-\psi^{+} t^{x} h_{n x}^{-}\right) \\
& +\left(\mathrm{Gh}_{\mathrm{n}, \chi}-\mathrm{G}_{\mathrm{n}, \chi} \mathrm{h}_{\mathrm{n}, \chi}\right)
\end{aligned}
$$




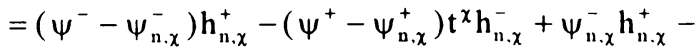

$$
\begin{aligned}
& -\psi_{n, x}^{+} t^{x} h_{n, x}^{-}-P_{n \cdot \chi}\left(\psi^{-} h_{n, x}^{+}-\psi^{+} t^{x} h_{n, x}^{-}\right)+ \\
& +\left(G h_{n, x}-G_{n, x} h_{n, x}\right)
\end{aligned}
$$

where

$$
\psi_{n, x}=\psi_{n, x}^{+}-\psi_{n, x}^{-} \quad, \quad\left(\psi_{n, x}^{+}(t)=\sum_{k=0}^{n-\chi} \alpha_{k} t^{k}, \quad \psi_{n, x}^{-}(t)=\sum_{k=-11}^{--\chi-1} \alpha_{k} t^{k}\right)
$$

is the polynomial of best uniform approximation to the function $\psi=\psi^{+}-\psi^{-}$with degree not exceeding $\mathrm{n}-\chi$ and $\psi_{\mathrm{n}, \chi}^{-} \mathrm{h}_{\mathrm{n}, \chi}^{+}-\psi_{\mathrm{n}, \chi}^{+} \mathrm{t}^{x} \mathrm{~h}_{\mathrm{v}, \chi}^{-}$is a polynomial of degree not exceeding $\mathrm{n}-\chi$. From (2.12) we have :

$$
L h_{n, \chi}-L_{n, \chi} h_{n, \chi}=\left(I-P_{n, \chi}\right)\left[\left(\psi^{-}-\psi_{n, \chi}^{-}\right) h_{n, \chi}^{+}-\left(\psi^{+}-\psi_{n, \chi}^{+}\right) t^{x} h_{n, \chi}^{-}\right]+\left(G h_{n, x}-G_{n, x} h_{n, x}\right) .
$$

From [3.8]:

$$
\left\|h_{n, x}^{ \pm}\right\|_{\varphi} \leq d_{1}\left\|h_{n, x}\right\|_{\varphi}
$$

and as was mentioned by Dzyedyk [1] and Gabdulkhaev [2] we obtain

$$
\left\|\left(\psi^{-}-\psi_{\mathrm{n}, \chi}^{-}\right) h_{n, \chi}^{+}-\left(\psi^{+}-\psi_{\mathrm{n}, \chi}^{+}\right) \mathrm{t}^{x} \mathrm{~h}_{\mathrm{n}, \chi}^{-}\right\|_{\varphi} \leq \mathrm{d}_{2} \varphi\left(\frac{1}{n-\chi}\right)\left\|\mathrm{h}_{\mathrm{n}, \chi}\right\|_{\varphi} .
$$

Then taking into accounts that $\left\|P_{n, \chi}\right\|_{\varphi} \leq d_{3} \ln (n-\chi)$, we obtain :

$$
\|\left(I-P_{n, \chi}\right)\left[\left(\psi^{-}-\psi_{n, \chi}^{-}\right) h_{n, \chi}^{+}-\left(\psi^{+}-\psi_{n, \chi}^{+}\right) t^{\chi} h_{n, \chi}^{-}\left\|_{\varphi} \leq\left(d_{4} \ln (n-\chi)\right) \varphi\left(\frac{1}{n-\chi}\right)\right\| h_{n, \chi} \|_{\varphi} .\right.
$$

Let $s_{n, x}(t)$ be the polynomial of best uniform approximation to the function

$$
s(t)=Q(t) \frac{c(y, t)}{\pi i} \int_{\gamma} H(t, \tau, y(\tau)) h_{n, \chi}(\tau) d \tau
$$

then we have

$$
\left\|s-s_{n, x}\right\|_{\varphi} \leq d_{s} \varphi\left(\frac{1}{n-\chi}\right)\left\|h_{n, \chi}\right\|
$$

and for arbitrary $h_{n, \chi} \in X_{n, \chi}$ we obtain

$$
\begin{aligned}
\left\|G h_{n, \chi}-G_{n, \chi} h_{n, \chi}\right\|_{\varphi} & =\left\|s-s_{n, \chi}+P_{n}\left(s_{n, \chi}-s\right)\right\|_{\varphi} \\
& \leq\left(d_{6} \ln (n-\chi)\right) \varphi\left(\frac{1}{n-\chi}\right)\left\|h_{n, \chi}\right\|_{\varphi} .
\end{aligned}
$$

Then from (2.13)-(2.15) we have

$$
\left\|\operatorname{Lh}_{n, x}-L_{n, x} h_{n, x}\right\|_{\varphi} \leq\left(d_{7} \ln (n-\chi)\right) \varphi\left(\frac{1}{n-\chi}\right)\left\|h_{n, x}\right\|_{\varphi} .
$$

Assume that there exists a linear bounded inverse operator $L_{0}^{-1}: H_{\varphi}(\gamma) \rightarrow \Omega_{\varphi}(\gamma)$. Since $\mathrm{L}_{\mathrm{o}} \mathrm{h}=\mathrm{Q}^{-1} \mathrm{Lh}$, then the operator $\mathrm{L}$ has a linear inverse, also from [2] and by virtue of $(2.16)$,(for sufficient large $\left.n, n \geq \max \left(n_{0}, \chi\right)\right)$, the operator $L_{n}$ has a linear inverse, moreover

$$
\left\|\mathrm{L}_{\mathrm{n}}^{-1}\right\| \leq\left\|\mathrm{L}^{-1}\right\|\left(1-\mathrm{e}_{\mathrm{n}}\right)^{-1}
$$

where

$$
e_{n}=\left\|L-L_{n}\right\| L^{-1} \|
$$

Now, for the right parts of (2.8) and (2.11) we have

$$
\left\|\mathrm{q}-\mathrm{q}_{\mathrm{n}}\right\|_{\varphi} \leq\left(\mathrm{d}_{8} \ln (\mathrm{n}-\chi)\right) \varphi\left(\frac{1}{\mathrm{n}-\chi}\right)\|\mathrm{f}\|_{\varphi} \leq \mathrm{d}_{9} \varphi\left(\frac{1}{\mathrm{n}-\chi}\right) \ln (\mathrm{n}-\chi) .
$$

Then by Theoreml.2 [2], and the inequalities (2.16)-(2.18) for the solution $\mathrm{h}^{*}$ of Equation (1.5) and 
the approximate solution $h_{n x}^{*}$ we obtain

$$
\left\|h^{*}-h_{n x}^{*}\right\|_{\varphi} \leq d_{10} \varphi\left(\frac{1}{n-\chi}\right) \ln (n-\chi)
$$

and the theorem is proved

From theorem 22 there exists the number

$$
\mathrm{n}_{n}=\min \left\{\mathrm{n} \in \mathbb{N} \quad \mathrm{e}_{\mathrm{n}}=\mathrm{d}_{11} \varphi\left(\frac{1}{\mathrm{n}-\chi}\right) \ln (\mathrm{n}-\chi)<1\right\},
$$

such that for arbitrary $n \geq \max \left(n_{0}, \chi\right)$ the $\operatorname{SLAE}(2.6)$ has the unique solution $h^{*}=\left(h_{-n}^{*} \ldots, h_{-x-L}^{*}, h_{0,}^{*} \ldots h_{n_{-x}}^{*}\right)$ and the following inequality is valid:

$$
\left\|y_{\mathrm{n} \chi}^{*}\left(\mathrm{~h}^{*}, .\right)-\mathrm{y}^{*}(.)\right\|_{\varphi} \leq \mathrm{d}_{12} \varphi\left(\frac{1}{\mathrm{n}-\chi}\right) \ln (\mathrm{n}-\chi)
$$

where $\mathrm{y}^{*} \in \Omega_{\varphi}(\gamma)$ is the unique solution of (2.7). Now, consider the operator

$$
L_{n}\left(y_{o}\right) h=\left(L_{o, n}\left(y_{o}\right) h, \ldots, L_{2(n-x), n}\left(y_{o}\right) h\right)
$$

where

$$
\begin{aligned}
L_{j, n}\left(y_{o}\right) h=a\left(y_{0}, t_{j}\right) y_{n, \chi}\left(h, t_{j}\right) & +\frac{b\left(y_{0}, t_{j}\right)}{\pi i} \int_{\gamma} \frac{y_{n, \chi}(h, \tau)}{\tau-t_{j}} d \tau+ \\
& +\frac{c\left(y_{0}, t\right)}{\pi i} \int_{\gamma} H\left(t_{j}, \tau, y_{0}\right) y_{n, \chi}(h, \tau) d \tau, \quad j=\overline{0,2(n-\chi)} .
\end{aligned}
$$

Then we obtain

$$
\begin{aligned}
& \left|L_{1,1}\left(y_{0}\right) h-P_{1,1}^{\prime}\left(\eta^{(1)}\right) h\right| \leq\left|a\left(y_{0}, t_{j}\right)-a\left(y_{n, x}\left(\eta^{(0)}, t_{j}\right), t_{j}\right)\right|\left|y_{n, \chi}\left(h, t_{1}\right)\right|+\left|b\left(y_{0}, t_{j}\right)-b\left(y_{n, \chi}\left(\eta^{(0)}, t_{1}\right), t_{1}\right)\right| \times \\
& \times\left|B y_{n, x}(h, \tau)\right|+\left|\alpha\left(y_{0}, t_{j}\right)-c\left(y_{1, x}\left(\eta^{(o)}, t_{j}\right), t_{j}\right) \| B H\left(t_{j}, \tau, y_{0}(\tau)\right) y_{n, x}(h, \tau)\right|+ \\
& +\left|B\left[H\left(t_{j}, \tau, y_{0}(\tau)\right)-H\left(t_{j}, \tau, y_{n, \chi}(h, \tau)\right)\right] y_{n, \chi}(h, \tau)\right|\left|\alpha\left(y_{n, x}\left(\eta^{(0)}, t_{j}\right), t_{j}\right)\right| .
\end{aligned}
$$

Taking into accounts that:

$$
\left\|y_{0}(.)-y_{n, \chi}\left(\eta^{(0)}, .\right)\right\|_{C(\gamma)} \leq d_{13} \varphi\left(\frac{1}{n-\chi}\right) \ln (n-\chi)
$$

we have

$$
\left\|L_{n}\left(y_{n}\right)-P_{n}^{\prime}\left(\eta^{(\circ)}\right)\right\|_{E_{\varphi}^{(1)} \rightarrow E_{\bullet}^{(2)}} \leq d_{14} \varphi\left(\frac{1}{n-\chi}\right) \ln (n-\chi) .
$$

Since for arbitrary $n \geq\left(n_{0}, \chi\right)$, there exists the bounded linear inverse operator $L_{n}^{-1}: E_{\varphi}^{(2)} \rightarrow E_{\varphi}^{(1)}$, then from (2.19) by Banach theorem it follows that there exists $n_{1} \geq \max \left(n_{0}, \chi\right)$ such that for arbitrary $n \geq n_{1}$, the linear operator $P_{j n}^{\prime}$ has bounded inverse, that is the SLAE (2.5) has the unique solution $\mathrm{h}^{*} \in \mathrm{E}_{\varphi}^{(1)}$ for arbitrary right sideg $=\mathrm{g}\left(\mathrm{t}_{\mathrm{j}}\right) \in \mathrm{E}_{\varphi}^{(2)}, \mathrm{j}=\overline{0,2(\mathrm{n}-\chi)}$.

Thus the following theorem is proved.

THEOREM 2.3. Let the coordinates of the vector $\eta^{(0)}=\left(\eta_{-n}^{(0)}, \ldots \eta_{-\chi-1}^{(0)}, \eta_{0}^{(0)}, \ldots \eta_{n-\chi}^{(0)}\right)$ be the Fourier coefficients of the function $y_{0} \in \Omega_{\varphi}(\gamma)$ and the conditions of Theorem 1.4 are satisfied and for $n \geq n_{1}$,

$$
\left\|\left[P_{n}^{\prime}\left(\eta^{(0)}\right)\right]^{-1}\right\|_{\varphi} \leq v_{2}^{\prime} \text { and }\left\|\left[P_{n}^{\prime}\left(\eta^{(0)}\right)\right]^{-1} P_{n}\left(\eta^{(0)}\right)\right\|_{\varphi} \leq \mu_{2}^{\prime}
$$

then, if $h_{2}^{\prime}=v_{2}^{\prime} M^{\prime} \mu_{2}^{\prime}<1 / 2$ and $r^{\prime} \geq r_{2}^{0^{\prime}}=\left(1-\sqrt{1-2 h_{2}^{\prime}}\right) \mu^{\prime}{ }_{2} / h^{\prime}{ }_{2}$ the SNAE (2.3) has the unique solution $\eta^{*}=\left(\eta_{-n}^{*}, \ldots, \eta_{-1-1}^{*}, \ldots, \eta_{b}^{*}, \ldots, \eta_{n-\chi}^{*}\right)$ in the sphere $\left(\eta^{(0)}, r_{2}^{0^{\prime}}\right)$ to which the following iteration process converges

$$
\eta^{(m+1)}=\eta^{(m)}-\left[P_{n}^{\prime}\left(\eta^{(o)}\right)\right]^{-1} P_{n}\left(\eta^{(m)}\right), \quad m=0,1 \ldots
$$

moreover the following mequality is true

$$
\left\|\eta^{(\mathrm{m})}-\eta^{*}\right\|_{\varphi} \leq\left(1-2 h_{2}^{\prime}\right)^{-1 / 2}\left(1-\sqrt{1-2 h_{2}^{\prime}}\right)^{m} \mu_{2}^{\prime} .
$$




\section{REFERENCES}

1. DZYEDYK, V.K. Introduction to the Theory of Uniform Approximation of Functions by Polynomıals, (in Russian), Nauka Moscow, 1977

2. GABDULKHAEV, B.G. Direct method for the solution of some functional equations, 1. IzV Vyss. Ucebn. Zaved Mat. Vol. 114, No. 11 (1971), P. 33-44.

3. Gakhov, F.D. Boundary Value Problems; Pergamon Press Ltd, 1966.

4. Gorlov, V.E. On the approximate solution of nonlinear Singular integral equations, lzv Vyss. Ucehn. Zaved Mat. Vol 167 No. 4 (1976), P. 122- 125.

5. GUSEINOV, A.I. and MUKHTAROV, KH.SH. Introduction to the Theory of Nonlinear Singular Integral Equations, Nauka Moscow, (in Russian) 1980.

6. MIKHLIN, S.G. and PROSSDORF, S. Singular Integral0peratorsAkademic-Verlag Berlin, 1986.

7. MUSAEV, B.I.; On approximate solution of the Singular integral equations; $\underline{A k a} . \underline{\text { Nauk. }} \underline{\text { Az. }} \underline{\text { SSR. }}$ Insitiute of Physics Preprint No. 17 (1986).

8. MUSKHELISHVILI, N.I. Singular Integral Equations, Noordhoff Groningen 1968.

9. POGORzelSKI, W. Integral Equations and Their Applications, Oxford, Pergman Press, Warsam: PWN-POl. Scient. Publ., 1966.

10. SALEH,M. H. and AMER,S. M. On a class of nonlinear singular integral equations with Cauchy hernel, AMSE Review Vol. 22 No. 1, (1992) P. 15-26.

11. VEKUA, I.N. On a Riemann boundary value problem, Trudy Tbilis. Mat. Inst. II (1942) I11- 116

12. WOLFERSDORF, L.V. A class of nonlinear Riemann-Hilbert problems for holomorphic function Math. Nachr. 116 (1984) 89-107. 


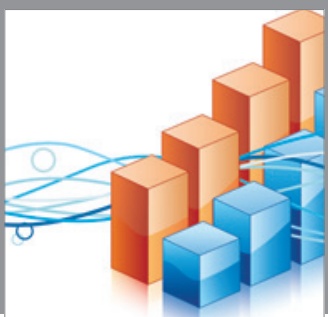

Advances in

Operations Research

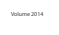

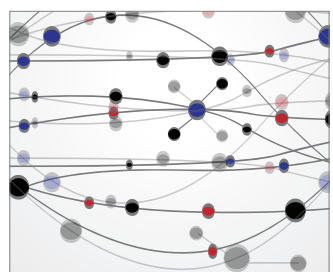

\section{The Scientific} World Journal
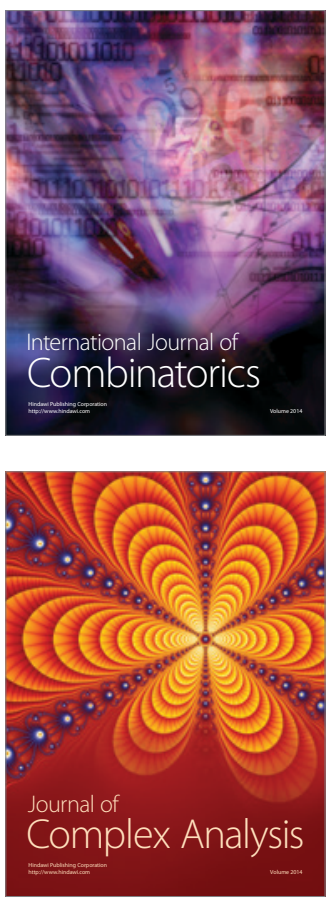

International Journal of

Mathematics and

Mathematical

Sciences
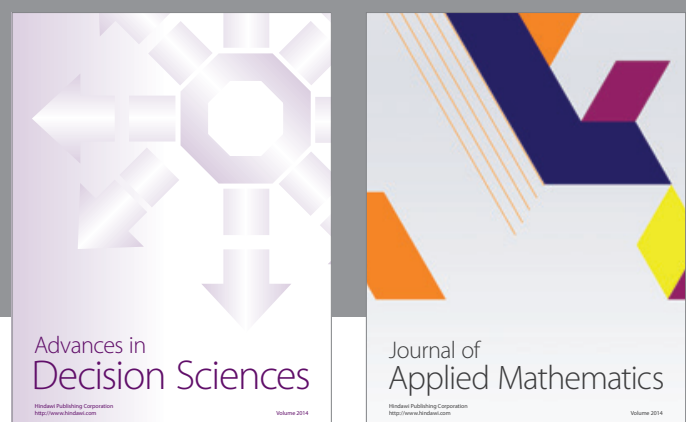

Journal of

Applied Mathematics
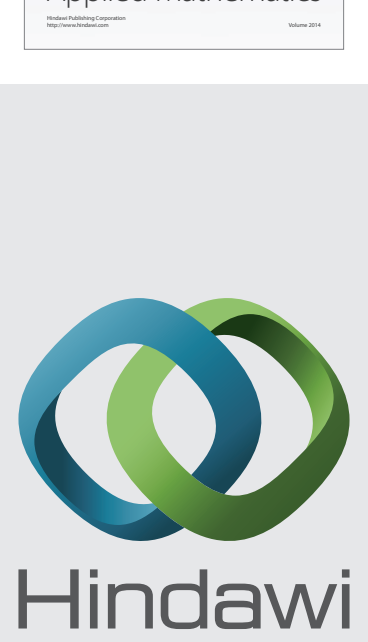

Submit your manuscripts at http://www.hindawi.com
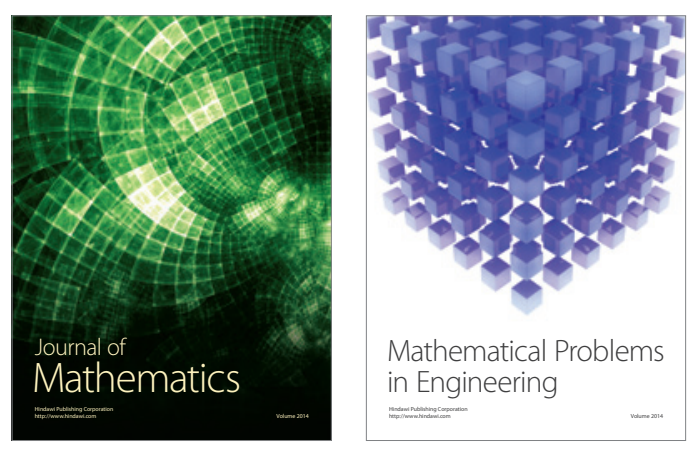

Mathematical Problems in Engineering
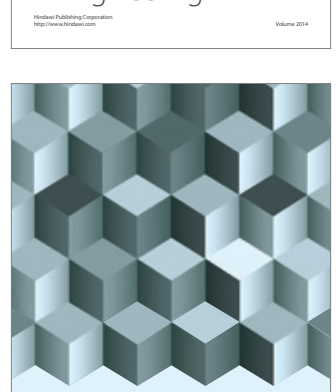

Journal of

Function Spaces
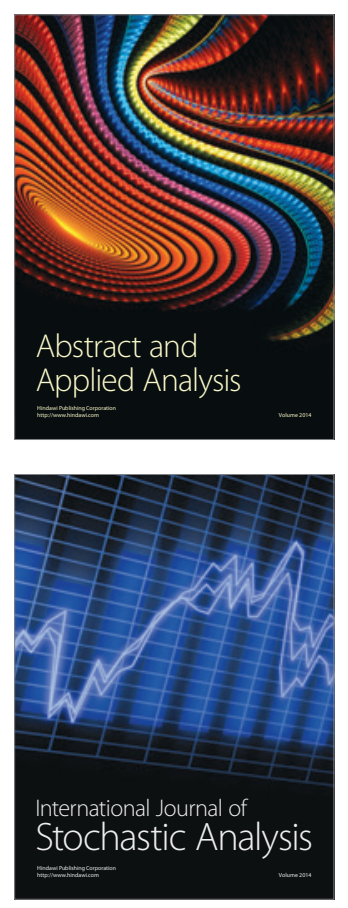

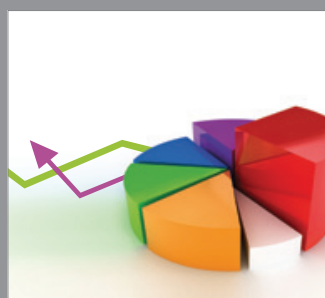

ournal of

Probability and Statistics

Promensencen
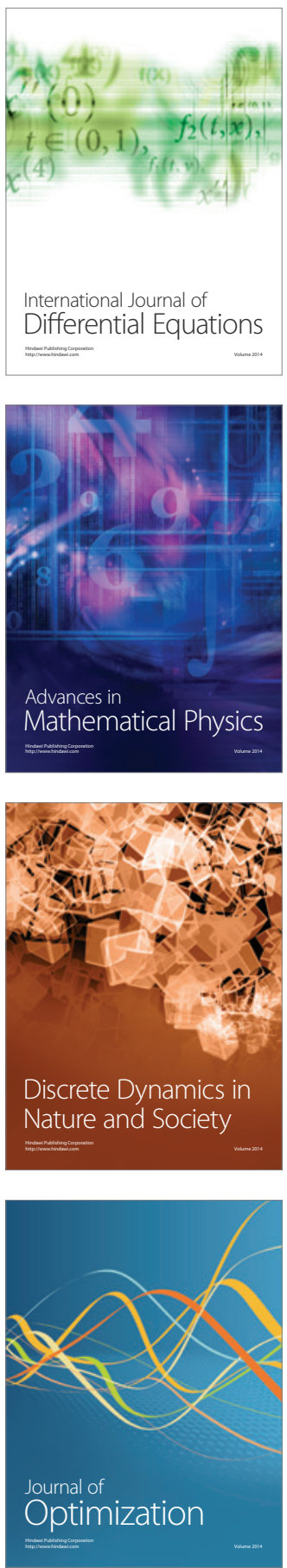